\title{
Natural Convection in a Vertical Microannulus With Superhydrophobic Slip and Temperature Jump
}

\author{
Chiu-On $\mathrm{Ng}^{1}$ \\ Department of Mechanical Engineering, The University of Hong Kong, \\ Pokfulam Road, Hong Kong \\ e-mail: cong@hku.hk \\ C. Y. Wang \\ Department of Mathematics, Michigan State University, \\ East Lansing, MI 48824
}

October 11, 2013

\begin{abstract}
Analytical solutions are derived for steady fully-developed buoyancy-driven flow in a vertical annular microchannel, of which either the inner or outer wall exhibits superhydrophobic velocity slip and temperature jump, and the inner wall is maintained either at constant wall temperature or constant heat flux. For the four possible cases of hydrodynamic and thermal boundary conditions, we determine the flow rate as a function of the core size, slip length and temperature jump coefficient. Asymptotic limits are obtained for very large slip and temperature jump. The effects of slip and temperature jump on two issues, namely, the optimum core radius for maximum flow rate and the singular increase of the flow rate for very small core radius, are investigated in particular.
\end{abstract}

Keywords: natural convection; superhydrophobic surface; temperature jump.

\footnotetext{
${ }^{1}$ Corresponding author.
} 


\section{Nomenclature}

$$
\begin{aligned}
& b=\text { normalized core radius } \\
& C=\text { dimensionless coefficient } \\
& g=\text { acceleration due to gravity, } \mathrm{m} / \mathrm{s}^{2} \\
& k=\text { thermal conductivity, } \mathrm{W} / \mathrm{m}^{2} \mathrm{~K} \\
& q=\text { heat flux, } \mathrm{W} / \mathrm{m} \\
& Q=\text { normalized flow rate } \\
& r=\text { normalized radial coordinate } \\
& \tilde{r}=\text { radial coordinate, } \mathrm{m} \\
& R=\text { radius of outer cylinder, } \mathrm{m} \\
& T=\text { temperature, } \mathrm{K} \\
& T_{0}=\text { ambient temperature, } \mathrm{K} \\
& T_{1}=\text { heated wall temperature, } \mathrm{K} \\
& w=\text { normalized axial velocity } \\
& \tilde{w}=\text { axial velocity, } \mathrm{m} / \mathrm{s} \\
& W=\text { velocity scale, } \mathrm{m} / \mathrm{s} \\
& \alpha=\text { dimensionless coefficient defined in Eq. (24) } \\
& \beta=\text { dimensionless coefficient defined in Eq. (23) } \\
& \beta_{t}=\text { thermal expansion coefficient, } 1 / \mathrm{K} \\
& \delta=\text { dimensionless coefficient defined in Eq. (12) } \\
& \gamma=\text { normalized temperature jump coefficient } \\
& \tilde{\gamma}=\text { temperature jump coefficient, } \mathrm{m} \\
& \lambda=\text { normalized slip length } \\
& \tilde{\lambda}=\text { slip length, } \mathrm{m} \\
& \Theta=\text { normalized temperature } \\
& \nu \quad=\text { kinematic viscosity, } \mathrm{m}^{2} / \mathrm{s}
\end{aligned}
$$




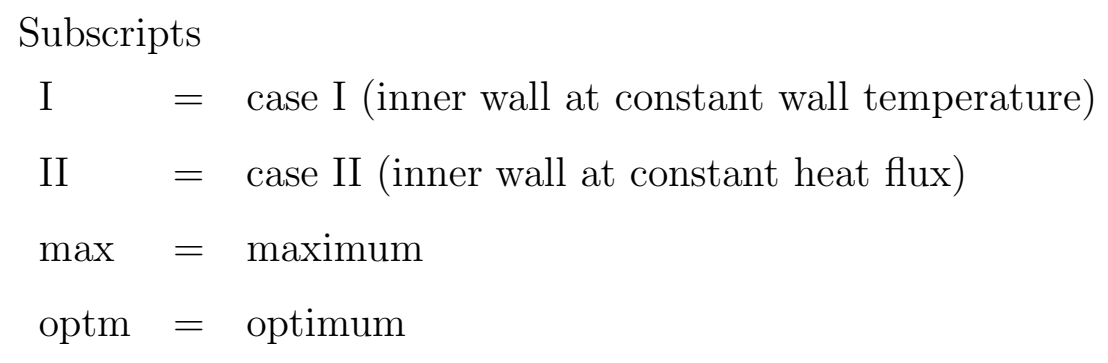

Superscripts
$\mathrm{A}=$ case $\mathrm{A}$ (inner wall being superhydrophobic surface)
$\mathrm{B}=$ case $\mathrm{B}$ (outer wall being superhydrophobic surface)

\section{Introduction}

An analytical study is presented in this paper on fully-developed natural convection of a liquid in an open-ended vertical annular microchannel with a heated core, where the hydrodynamic and thermal boundary conditions are different on the inner and outer walls of the channel. One of the two walls is a superhydrophobic surface exhibiting velocity slip and temperature jump, while the other wall is a normal surface without slip and temperature jump. Cases in which the inner wall is maintained either at constant wall temperature or constant heat flux, while the outer wall is always maintained at ambient temperature, are investigated. As free convection can transport fluid without the need of any mechanical pumping, this mechanism is expected to be of great potential use in microfluidics. Since superhydrophobicity is increasingly applied to drag reduction in microchannel flow, it is of fundamental interest to find out how free convection may happen in a channel with velocity slip and temperature temperature.

This paper aims to extend the work by Wang [1], who studied a similar problem of natural convection in an annular duct but without considering velocity slip and temperature jump. Wang [1] found that, under each heating condition, there is an optimum size of the channel core for maximal flow and energy transport. He also investigated the singular behavior, in the form of a sharp rise from zero, of the flow when the core is very small. We shall determine in this study how the maximum flow rate and the corresponding optimum core size will change as a function of the slip and temperature jump, for each of the heating and surface conditions. We shall also examine how the slip and temperature jump will interplay with the singular 
behavior to determine the maximum flow rate under some particular conditions.

For channels with two distinct boundaries, the thermal conditions on each boundary may be different. For fully-developed natural convection between two parallel plates, the solution is well known (e.g., Jaluria [2]). Joshi [3] analytically solved the problem for fullydeveloped laminar natural convection in a vertical annulus under isothermal wall boundary conditions. El-Shaarawi and Al-Nimr [4] further developed solutions for several combinations of boundary conditions. Extensions to vertical transient annular flow [5, 6], and flow in eccentric annuli $[7,8]$ were made. Velocity slip and temperature jump are not taken into account in these studies.

In the context of rarefied gas flow, natural convection in a vertical microannulus with velocity slip and temperature jump has been studied by Weng and Chen [9]. Extension to mixed convection was made by Avci and Aydin [10]. The problems of optimal core size and singular rise of flow rate were, however, not studied by these authors. Buonomo and Manca [11] performed a numerical study on developing natural convection flow of air in a vertical parallel-plate microchannel under slip and jump boundary conditions. In these works, slip and jump are considered to result from rarefaction effect, which is essentially a property of the fluid. Hence, in their problems, the two walls have the same slip and temperature jump coefficients.

In this study, we consider hydrodynamic and thermal slip arising from superhydrophobicity of a surface. Unlike gas slip, superhydrophobic slip is essentially due to wall characteristics, such as microstructures with tiny grooves or cavities filled with a gas [12]. In view of the fact that superhydrophobic surfaces are expensive to fabricate, it is desirable, from a cost-effect point of view, that only one wall of a microchannel is a superhydrophobic surface, and the other wall is a normal surface without slip. This study aims to investigate natural convection in such a vertical microannulus, of which either the inner or outer wall is a superhydrophobic surface. It is anticipated that the flow will depend on whether heat is added under constant wall temperature or constant heat flux condition, and whether the superhydrophobic surface is on the hotter or cooler wall of the channel. We aim to find out in this work how these factors will affect free convection in a microannulus, which is a problem of interest because of the existence of an optimum core size for maximum flow rate, and the possible singular 
behavior of the flow rate when the core is very small.

This paper is organized as follows. In Section 2, the case where heating is applied to the core by uniform wall temperature is considered. On using the Boussinesq approximation, analytical solutions are derived for the temperature and velocity distributions, for the subcases where the superhydrophobic surface is either on the inner or outer wall of the annular duct. The flow rate is found as a function of the core radius, the velocity slip length, and the temperature jump coefficient. Limits for the flow rate corresponding to zero or very large slip and temperature jump are deduced. In Section 3, the same problem is solved again, but under the condition that the core is heated by uniform heat flux. In Section 4, we present results to illustrate how the flow rate is affected in different manners by the three controlling parameters, depending on the thermal and hydrodynamic boundary conditions. To harvest the advantage of superhydrophobicity, it is desirable to have the cooler outer wall be the superhydrophobic surface. We shall show that the maximum flow rate may increase without bound with the temperature jump if the jump is on the outer boundary of the channel. If the core is maintained at constant wall temperature, the maximum flow rate occurs at a very small core radius (it may even occur right at the top of the singular rise), which may not be very practical to achieve. If the core is maintained at constant heat flux, the maximum flow rate occurs at a finite value of the core radius (in the range of $15-30 \%$ of the outer radius), which is much more readily to achieve.

\section{Case I: Heating by Uniform Wall Temperature}

Consider steady fully-developed free convection in an open vertical annular duct, composed of a cylindrical core of radius $b R$, and an outer cylinder of radius $R$, where $0<b<1$ is the ratio of the two radii. The outer wall is maintained at ambient temperature $T_{0}$, and the inner wall is uniformly heated to a higher temperature $T_{1}$. See Fig. 1 for a definition sketch of the problem. We consider separately the cases in which either the inner or outer wall is a superhydrophobic (SH) surface with effective slip length $\tilde{\lambda}$ and temperature jump coefficient $\tilde{\gamma}$, while the other wall is a normal no-slip surface. Given long enough length of the channel, a thermally fully-developed region is established, where the temperature $T(\tilde{r})$ is independent 
of vertical position. The flow is fully developed as well, with an upward velocity $\tilde{w}(\tilde{r})$, where $\tilde{r}$ is the radial coordinate. Assuming that there is no forced convection, the dynamic pressure gradient is zero.

Using the Boussinesq approximation, the momentum and energy equations are as follows:

$$
\begin{gathered}
g \beta_{t}\left(T-T_{0}\right)+\nu \nabla^{2} \tilde{w}=0 \\
\nabla^{2} T=0
\end{gathered}
$$

where $g$ is the acceleration due to gravity, $\beta_{t}$ is the thermal expansion coefficient, and $\nu$ is the kinematic viscosity of the fluid.

Let us introduce the following dimensionless variables:

$$
w=\tilde{w} / W, \quad \Theta=\left(T-T_{0}\right) /\left(T_{1}-T_{0}\right), \quad(r, \lambda, \gamma)=(\tilde{r}, \tilde{\lambda}, \tilde{\gamma}) / R
$$

where $W=g \beta_{t}\left(T_{1}-T_{0}\right) R^{2} / \nu$ is the characteristic velocity for the flow, and $\lambda$ and $\gamma$ are respectively the normalized slip length and normalized temperature jump coefficient. In terms of these dimensionless variables, the momentum and energy equations become

$$
\begin{gathered}
\Theta+\frac{1}{r} \frac{\mathrm{d}}{\mathrm{d} r}\left(r \frac{\mathrm{d} w}{\mathrm{~d} r}\right)=0 \\
\frac{1}{r} \frac{\mathrm{d}}{\mathrm{d} r}\left(r \frac{\mathrm{d} \Theta}{\mathrm{d} r}\right)=0
\end{gathered}
$$

\subsection{Case I(A): Inner Wall Being Superhydrophobic Surface}

We first consider the case where the inner wall is a $\mathrm{SH}$ surface heated to temperature $T_{1}$, and the outer wall is a no-slip surface at ambient temperature $T_{0}$. The boundary conditions are accordingly as follows.

At the $\mathrm{SH}$ surface $(r=b)$, the temperature jump condition is

$$
T-T_{1}=\tilde{\gamma} T^{\prime} \Rightarrow \Theta(b)-1=\gamma \Theta^{\prime}(b),
$$

and the partial-slip condition is

$$
w(b)=\lambda w^{\prime}(b) .
$$


At the no-slip surface $(r=1)$, the conditions are simply

$$
T=T_{0} \Rightarrow \Theta(1)=0, \quad w(1)=0 .
$$

The solutions to Eqs. (4) and (5) satisfying these boundary conditions, distinguished by the subscript "I" and superscript "A", are as follows:

$$
\begin{gathered}
\Theta_{\mathrm{I}}^{\mathrm{A}}(r)=C_{\mathrm{I}}^{\mathrm{A}} \ln r, \\
w_{\mathrm{I}}^{\mathrm{A}}(r)=\frac{C_{\mathrm{I}}^{\mathrm{A}}}{4}\left[-r^{2} \ln r+r^{2}+\delta \ln r-1\right],
\end{gathered}
$$

where

$$
\begin{gathered}
C_{\mathrm{I}}^{\mathrm{A}}=\frac{b}{b \ln b-\gamma}, \\
\delta=\frac{b\left[b(b-2 \lambda) \ln b+1-b^{2}+b \lambda\right]}{b \ln b-\lambda} .
\end{gathered}
$$

The volume flow rate is then given by

$$
\begin{aligned}
Q_{\mathrm{I}}^{\mathrm{A}}(b, \lambda, \gamma) & =2 \pi \int_{b}^{1} w_{\mathrm{I}}^{\mathrm{A}} r \mathrm{~d} r \\
& =\frac{C_{\mathrm{I}}^{\mathrm{A}} \pi}{32}\left\{b^{2}\left[4\left(b^{2}-2 \delta\right) \ln b+4 \delta-5 b^{2}+8\right]-4 \delta-3\right\} .
\end{aligned}
$$

We may check that when both walls are no-slip surfaces, i.e., on the vanishing of both velocity slip and temperature jump: $\lambda=\gamma=0$, the flow rate reduces to the one previously obtained by Wang [1]:

$$
Q_{\mathrm{I}}^{\mathrm{A}}(b, 0,0)=\frac{\pi}{32(\ln b)^{2}}\left\{-\ln b\left[4 b^{4} \ln b+\left(3+7 b^{2}\right)\left(1-b^{2}\right)\right]-4\left(1-b^{2}\right)^{2}\right\},
$$

which, as noted by Wang [1], exhibits singular behavior for small $b$ :

$$
Q_{\mathrm{I}}^{\mathrm{A}}\left(b \rightarrow 0^{+}, 0,0\right) \sim-\frac{3 \pi}{32 \ln b} .
$$

At the other extreme, when both velocity slip and temperature jump are very large: $\lambda \gg 1$, $\gamma \gg 1$, the flow rate has the following asymptotic limit:

$$
\begin{aligned}
Q_{\mathrm{I}}^{\mathrm{A}}(b, \lambda \gg 1, \gamma \gg 1) \sim & \frac{\pi b}{32 \gamma}\left\{-4 b^{2} \ln b\left[-4 b^{2} \ln b+5 b^{2}-2\right]\right. \\
& \left.+3\left(1-3 b^{2}\right)\left(1-b^{2}\right)\right\}
\end{aligned}
$$

which is noted to be (i) independent of the slip length $\lambda$, (ii) decreasing with increasing temperature jump according to $\gamma^{-1}$, and (iii) regular as $b \rightarrow 0$. One can show that the singular behavior of $Q_{\mathrm{I}}^{\mathrm{A}}\left(b \rightarrow 0^{+}\right)$is removed by a non-zero $\gamma$. 


\subsection{Case I(B): Outer Wall Being Superhydrophobic Surface}

We then consider the case where the inner wall is a no-slip surface heated to $T_{1}$, and the outer surface is a $\mathrm{SH}$ surface at ambient temperature $T_{0}$. The boundary conditions are accordingly as follows.

At the no-slip surface $(r=b)$, the conditions are

$$
T=T_{1} \Rightarrow \Theta(b)=1, \quad w(b)=0
$$

At the SH surface $(r=1)$, the temperature jump condition is

$$
T-T_{0}=-\tilde{\gamma} T^{\prime} \Rightarrow \Theta(1)=-\gamma \Theta^{\prime}(1)
$$

and the partial-slip condition is

$$
w(1)=-\lambda w^{\prime}(1)
$$

The solutions to Eqs. (4) and (5) satisfying these boundary conditions, distinguished by the subscript "I" and superscript "B", are as follows:

$$
\begin{gathered}
\Theta_{\mathrm{I}}^{\mathrm{B}}(r)=C_{\mathrm{I}}^{\mathrm{B}}(\ln r-\gamma), \\
w_{\mathrm{I}}^{\mathrm{B}}(r)=\frac{C_{\mathrm{I}}^{\mathrm{B}}}{4}\left[-r^{2} \ln r+(1+\gamma) r^{2}+\beta \ln r+\alpha\right],
\end{gathered}
$$

where

$$
\begin{gathered}
C_{\mathrm{I}}^{\mathrm{B}}=\frac{1}{\ln b-\gamma}, \\
\beta=\frac{b^{2} \ln b-b^{2}(1+\gamma)+\gamma+\lambda+2 \gamma \lambda+1}{\ln b-\lambda}, \\
\alpha=\frac{-\left(b^{2} \lambda+\gamma+\lambda+2 \gamma \lambda+1\right) \ln b+b^{2}(1+\gamma) \lambda}{\ln b-\lambda} .
\end{gathered}
$$

The volume flow rate is then given by

$$
\begin{aligned}
Q_{\mathrm{I}}^{\mathrm{B}}(b, \lambda, \gamma) & =2 \pi \int_{b}^{1} w_{\mathrm{I}}^{\mathrm{B}} r \mathrm{~d} r \\
& =\frac{C_{\mathrm{I}}^{\mathrm{B}} \pi}{32}\left\{4 b^{2}\left(b^{2}-2 \beta\right) \ln b+\left(1-b^{2}\right)\left[\left(1+b^{2}\right)(5+4 \gamma)-4 \beta+8 \alpha\right]\right\} .
\end{aligned}
$$

Again, for $\lambda=\gamma=0$, we may check that $Q_{\mathrm{I}}^{\mathrm{B}}(b, 0,0)=Q_{\mathrm{I}}^{\mathrm{A}}(b, 0,0)$, which as given in Eq. (14) is the flow rate previously deduced by Wang [1]. For $\lambda \gg 1$ and $\gamma \gg 1$, the following asymptotic limit can be obtained:

$$
Q_{\mathrm{I}}^{\mathrm{B}}(b, \lambda \gg 1, \gamma \gg 1) \sim-\frac{\pi \gamma \lambda\left[4 \ln b+\left(3-b^{2}\right)\left(1-b^{2}\right)\right]}{8(\ln b-\gamma)(\ln b-\lambda)} \quad(\text { for any } b),
$$


which is always singular near $b=0$. In fact, the singular behavior of $Q_{\mathrm{I}}^{\mathrm{B}}\left(b \rightarrow 0^{+}\right)$cannot be eliminated by any values of $\lambda$ and $\gamma$. Also note that for $b$ not close to zero such that $|\ln b|$ is much less than both $\lambda$ and $\gamma$, the above limit reduces to

$$
Q_{\mathrm{I}}^{\mathrm{B}}(b, \lambda \gg 1, \gamma \gg 1) \sim-\frac{\pi}{8}\left[4 \ln b+\left(3-b^{2}\right)\left(1-b^{2}\right)\right] \quad(\text { for } b \text { not close to zero })
$$

which is independent of both $\lambda$ and $\gamma$.

\section{Case II: Heating by Uniform Heat Flux}

Consider the same problem as formulated in case I, except that the heating is now due to a constant heat flux $q$ applied to the inner wall, while the outer wall is maintained at ambient temperature $T_{0}$. The dimensionless momentum and energy equations are still given by Eqs. (4) and (5), but the normalization is now changed to

$$
w=\tilde{w} / W, \quad \Theta=\left(T-T_{0}\right) /(q R / k),
$$

where $W=g \beta_{t} q R^{3} / \nu k$ is the characteristic velocity for the flow, and $k$ is the thermal conductivity of the fluid.

\subsection{Case II(A): Inner Wall Being Superhydrophobic Surface}

We first consider the case where the inner wall is a SH surface under the uniform heat flux condition, and the outer wall is a no-slip surface at ambient temperature. The boundary conditions are accordingly as follows.

At the SH surface $(r=b)$, the temperature jump condition is

$$
q=-k T^{\prime} \Rightarrow \Theta^{\prime}(b)=-1
$$

and the partial-slip condition is

$$
w(b)=\lambda w^{\prime}(b)
$$

At the no-slip surface $(r=1)$, the conditions are simply

$$
T=T_{0} \Rightarrow \Theta(1)=0, \quad w(1)=0 .
$$


The solutions to Eqs. (4) and (5) satisfying these boundary conditions, distinguished by the subscript "II" and superscript "A", are as follows:

$$
\begin{gathered}
\Theta_{\mathrm{II}}^{\mathrm{A}}(r)=-b \ln r, \\
w_{\mathrm{II}}^{\mathrm{A}}(r)=-\frac{b}{4}\left[-r^{2} \ln r+r^{2}+\delta \ln r-1\right],
\end{gathered}
$$

where $\delta$ is given by Eq. (12). The volume flow rate is then given by

$$
Q_{\mathrm{II}}^{\mathrm{A}}(b, \lambda)=2 \pi \int_{b}^{1} w_{\mathrm{II}}^{\mathrm{A}} r \mathrm{~d} r=-\frac{b \pi}{32}\left\{b^{2}\left[4\left(b^{2}-2 \delta\right) \ln b+4 \delta-5 b^{2}+8\right]-4 \delta-3\right\},
$$

which is independent of $\gamma$. From Eqs. (13) and (34),

$$
Q_{\mathrm{II}}^{\mathrm{A}}=(-b \ln b+\gamma) Q_{\mathrm{I}}^{\mathrm{A}}
$$

For $\lambda=0$,

$$
Q_{\mathrm{II}}^{\mathrm{A}}(b, 0)=-\frac{\pi b}{32 \ln b}\left\{-\ln b\left[4 b^{4} \ln b+\left(3+7 b^{2}\right)\left(1-b^{2}\right)\right]-4\left(1-b^{2}\right)^{2}\right\},
$$

which is also previously obtained by Wang [1]. For $\lambda \gg 1$, the flow rate tends to the following asymptotic limit:

$$
Q_{\mathrm{II}}^{\mathrm{A}}(b, \lambda \gg 1) \sim \frac{\pi b}{32}\left\{-4 b^{2} \ln b\left[-4 b^{2} \ln b+5 b^{2}-2\right]+3\left(1-3 b^{2}\right)\left(1-b^{2}\right)\right\}
$$

which is independent of both $\lambda$ and $\gamma$, and regular near $b=0$. In fact, for any $\lambda$,

$$
Q_{\mathrm{II}}^{\mathrm{A}}\left(b \rightarrow 0^{+}, \lambda\right) \sim \frac{3 \pi b}{32}
$$

\subsection{Case II(B): Outer Wall Being Superhydrophobic Surface}

We then consider the case where the inner wall is a no-slip surface under the uniform heat flux condition, and the outer surface is a SH surface at ambient temperature. The boundary conditions are accordingly as follows.

At the no-slip surface $(r=b)$, the conditions are

$$
q=-k T^{\prime} \Rightarrow \Theta^{\prime}(b)=-1, \quad w(b)=0 .
$$


At the SH surface $(r=1)$, the temperature jump condition is

$$
T-T_{0}=-\tilde{\gamma} T^{\prime} \Rightarrow \Theta(1)=-\gamma \Theta^{\prime}(1)
$$

and the partial-slip condition is

$$
w(1)=-\lambda w^{\prime}(1)
$$

The solutions to Eqs. (4) and (5) satisfying these boundary conditions, distinguished by the subscript "II" and superscript "B", are as follows:

$$
\begin{gathered}
\Theta_{\mathrm{II}}^{\mathrm{B}}(r)=-b(\ln r-\gamma), \\
w_{\mathrm{II}}^{\mathrm{B}}(r)=-\frac{b}{4}\left[-r^{2} \ln r+(1+\gamma) r^{2}+\beta \ln r+\alpha\right],
\end{gathered}
$$

where $\beta$ and $\alpha$ are given by Eqs. (23) and (24), respectively. The volume flow rate is then given by

$$
\begin{aligned}
Q_{\mathrm{II}}^{\mathrm{B}}(b, \lambda, \gamma) & =2 \pi \int_{b}^{1} w_{\mathrm{II}}^{\mathrm{B}} r \mathrm{~d} r \\
& =-\frac{b \pi}{32}\left\{4 b^{2}\left(b^{2}-2 \beta\right) \ln b+\left(1-b^{2}\right)\left[\left(1+b^{2}\right)(5+4 \gamma)-4 \beta+8 \alpha\right]\right\}
\end{aligned}
$$

From Eqs. (25) and (44),

$$
Q_{\mathrm{II}}^{\mathrm{B}}=(-b \ln b+b \gamma) Q_{\mathrm{I}}^{\mathrm{B}}
$$

For $\lambda=\gamma=0$, we may check that $Q_{\mathrm{II}}^{\mathrm{B}}(b, 0,0)=Q_{\mathrm{II}}^{\mathrm{A}}(b, 0)$, which is given in Eq. (36). For $\lambda \gg 1$ and $\gamma \gg 1$, the flow rate tends to the following asymptotic limit:

$$
Q_{\mathrm{II}}^{\mathrm{B}}(b, \lambda \gg 1, \gamma \gg 1) \sim \frac{\pi \gamma \lambda b\left[4 \ln b+\left(3-b^{2}\right)\left(1-b^{2}\right)\right]}{8(\ln b-\lambda)},
$$

which is regular near $b=0$ :

$$
Q_{\mathrm{II}}^{\mathrm{B}}\left(b \rightarrow 0^{+}, \lambda \gg 1, \gamma \gg 1\right) \sim \frac{\pi \gamma \lambda b \ln b}{2(\ln b-\lambda)} .
$$

\section{Discussion}

We show in Fig. 2 the flow rates $Q_{\mathrm{I}}^{\mathrm{A}}$ and $Q_{\mathrm{I}}^{\mathrm{B}}$ as functions of $\lambda$ and $\gamma$, where the heating is due to uniform wall temperature, and $b=0.1$. Increasing the slip length $\lambda$ can increase the flow 
rate when either the inner or outer wall is the SH surface, as expected. However, increasing the temperature jump coefficient $\gamma$ will have opposite effects on the flow when the SH surface is on the inner or outer wall of the channel. When the SH surface is on the inner wall (case A), the temperature jump of the surface itself will impede the heat transfer into the channel, and therefore lower the temperature rise or buoyancy in the fluid. The temperature jump will, therefore, have a negative (i.e., suppressing) effect on the flow when the heating is applied to the SH surface, or when the temperature decreases away from the SH surface. In contrast, when the SH surface is on the outer wall (case B), the temperature jump of the SH surface is to impede heat loss through the outer wall, and will therefore increase the bulk temperature rise or buoyancy in the fluid. Consequently, the temperature jump will have a positive (i.e., enhancing) effect on the natural convection when the temperature decreases toward the $\mathrm{SH}$ surface.

We then show in Fig. 3 the flow rates $Q_{\mathrm{II}}^{\mathrm{A}}$ and $Q_{\mathrm{II}}^{\mathrm{B}}$ as functions of $\lambda$ and $\gamma$, where the heating is due to uniform wall flux, and $b=0.2$. In this case, the temperature jump coefficient will have no effect on the flow when the heated inner wall is the SH surface. This is because the temperature jump will cause a discontinuity of temperature, but not heat flux, across the fluid-wall interface. This explains why $Q_{\mathrm{II}}^{\mathrm{A}}$ is independent of $\gamma$. If the SH surface is on the outer wall, the temperature jump of the surface will have a positive effect on the flow, for the same reason as explained above for case I.

The flow rates for the four cases as functions of the core radius $b$ are shown in Fig. 4, where $(\lambda, \gamma)=(0,0),(1,0),(0,1),(1,1)$. In the lower limit $b=0$, the flow rates are zero because of the diminishing strength of the heat source. In the upper limit $b=1$, the flow rates are also zero because of the vanishing sectional area of the annulus. There must exist for each case an intermediate value of $b$, which is referred to as the optimum core radius $b_{\text {optm, at }}$ which the flow rate is the maximum. The maximum flow rates $Q_{\max }$ and the corresponding $b_{\text {optm }}$ for the cases shown in Fig. 4 are provided in Table 1. These optimum values, which are obtained with the aid of Mathematica, can serve as benchmarks for approximate or numerical methods solving similar problems in the future. The optimum values for the limiting cases of $(\lambda, \gamma)=(0,0)$, given by Eqs. (14) and (36), agree with those reported by Wang [1]. Wang [1] also pointed out the singular behavior when $b \rightarrow 0$, which explains the sharp rise of the 
curves near $b=0$ under the uniform wall temperature condition. The singular behavior is essentially due to the term $\ln b$ in the denominator of the flow rate expressions. The singular behavior of $Q_{\mathrm{I}}^{\mathrm{A}}$ near $b=0$ is eliminated, however, by a non-zero $\gamma$, as is seen in Fig. 4(a). The singular behavior of $Q_{\mathrm{I}}^{\mathrm{B}}$ near $b=0$ is persistent, irrespective of the values of $\lambda$ and $\gamma$, as is seen in Fig. 4(b).

A magnified view of the singular rise of the flow rate in case $\mathrm{I}(\mathrm{B})$ is illustrated in Fig. 5 , which shows the cases $(\lambda, \gamma)=(0,0),(10,0),(0,10),(10,10)$. Values of $Q_{\max }\left(b_{\mathrm{optm}}\right)$ for these cases are also provided in Table 1. For further illustration, we also include the values for the cases $(\lambda, \gamma)=(50,0),(0,50),(50,50)$ in Table 1 . The maximum flow rate increases, while the optimum core radius can diminish significantly with increase of $\lambda$ or $\gamma$. This results in a very dramatic sharp rise of the flow rate, virtually reaching the maximum at the top of the rise, for large slip or temperature jump.

The maximum flow rate and the corresponding optimum core radius will change as the velocity slip and temperature jump change, in a manner depending on the heating and the surface conditions. Figure 6 shows, for the four cases, $Q_{\max }$ and $b_{\text {optm }}$ as functions of $\lambda$ and $\gamma$, where $\lambda=\gamma$. Increasing the slip and temperature jump equally will decrease $Q_{\max }$ in case I(A) (inner surface being SH surface under uniform wall temperature), but will increase $Q_{\max }$ in all other cases. To explain the behaviors, let us recall the asymptotic limits that we have deduced for the flow rates under the condition of very large $\lambda$ and $\gamma$. First, we get from Eq. (16) that $Q_{\mathrm{I}}^{\mathrm{A}}$ will, asymptotically, become independent of the velocity slip, but decrease with increasing temperature jump according to $\gamma^{-1}$. This explains the decline of $Q_{\mathrm{I}, \max }^{\mathrm{A}}$ with increasing $\gamma$. Second, as discussed above, $Q_{\mathrm{I}}^{\mathrm{B}}$ is always singular near $b=0$, and the optimum core radius in case $\mathrm{I}(\mathrm{B})$ will be increasingly close to zero, $b_{\text {optm }} \sim 0^{+}$, as $\lambda$ and $\gamma$ increase. This is clearly shown in Fig. 6(b). By maximizing the flow rate given in Eq. (26), we may obtain the optimum core radius as given by

$$
\ln b_{\mathrm{optm}}=-\frac{3+\sqrt{(4 \gamma+3)(4 \lambda+3)}}{4} \quad(\text { for case } \mathrm{I}(\mathrm{B}), \lambda \gg 1, \gamma \gg 1)
$$

by which the maximum flow rate is approximately equal to

$$
Q_{\mathrm{I}}^{\mathrm{B}}\left(b_{\mathrm{optm}}, \lambda \gg 1, \gamma \gg 1\right) \sim \frac{\pi \gamma \lambda \sqrt{\gamma \lambda}}{2(\sqrt{\gamma \lambda}+\gamma)(\sqrt{\gamma \lambda}+\lambda)}
$$


which will increase monotonically with increase in either $\lambda$ or $\gamma$. In particular, when $\gamma=\lambda$, the right-hand side of the equation above reduces to $\pi \gamma / 8$. This explains the linear increase of $Q_{\mathrm{I}, \max }^{\mathrm{B}}$ with increasing $\gamma$, as shown in Fig. 6(a). Note that $b_{\mathrm{optm}}$ as given by Eq. (48) is indeed infinitesimally small. Only near $b=b_{\mathrm{optm}} \sim 0^{+}$will $Q_{\mathrm{I}}^{\mathrm{B}}$ increase with $\lambda$ or $\gamma$ without saturation. For $b$ that is not close to zero, $Q_{\mathrm{I}}^{\mathrm{B}}$ will become independent of $\lambda$ and $\gamma$ when these two parameters are very large, as is bounded by the asymptote given in Eq. (27). Third, Eq. (37) shows that, for $\lambda \gg 1, Q_{\mathrm{II}}^{\mathrm{A}}$ is essentially independent of both the velocity slip and temperature jump. This explains why $Q_{\mathrm{II}, \max }^{\mathrm{A}}$ tends to a constant as $\lambda$ increases, as shown in

Fig. 6(a). Fourth, we get from Eq. (46) that $Q_{\mathrm{II}}^{\mathrm{B}}$ is affected by the slip and temperature jump through the factor $\gamma \lambda /(\ln b-\lambda)$, which is increasingly proportional to $\gamma$ as $\lambda$ increases. This explains the approximately linear increase of $Q_{\mathrm{II}, \max }^{\mathrm{B}}$ with increasing $\gamma$, as shown in Fig. 6(a).

\section{Conclusions}

We have developed analytical solutions for free convection in an open vertical annular duct with a heated core. The core is heated with its wall under constant temperature (case I) or constant heat flux (case II) condition. Either the inner wall (case A) or the outer wall (case B) is a superhydrophobic surface with an effective velocity slip and temperature jump. The following conclusions can be drawn from the present study.

1. When the inner wall is under the constant temperature condition, the flow rate may rise singularly as the core radius is increased from zero. Such singular behavior is removed by a temperature jump on the inner wall, but it will persist if the temperature jump is on the outer wall.

2. The optimum core radius for maximum flow rate is in the range of $0.1<b_{\text {optm }}<0.3$ for any values of slip and temperature jump in cases $\mathrm{I}(\mathrm{A}), \mathrm{II}(\mathrm{A})$ and $\mathrm{II}(\mathrm{B})$. In these cases, the flow rate attains its maximum in a smooth manner. In case $\mathrm{I}(\mathrm{B})$, the optimum core radius will decrease from $b_{\mathrm{optm}} \approx 0.1$ to infinitesimally small as the slip or the temperature jump increases. In this case, for large slip or temperature jump, the flow rate will increase sharply from zero to the maximum as the core radius barely increases from zero. 
3. Increasing the slip length, when it is small, will increase the flow rate in all cases. The flow rate will, however, become increasingly independent of the slip length when the slip length itself gets very large (i.e., toward the perfect-slip limit). The only exception occurs in case $\mathrm{I}(\mathrm{B})$ : when the core radius is at or very close to the optimum value, the flow rate may increase with the slip without saturation. The optimum radius will, however, diminish to infinitesimally small when the slip length is large.

4. The flow is unaffected by the temperature jump in case $\operatorname{II}(\mathrm{A})$, but is negatively affected by the temperature jump in case $\mathrm{I}(\mathrm{A})$, where heat transfer from the core into the duct is impeded by the temperature jump. In case $\mathrm{I}(\mathrm{A})$, the flow rate is always decreased by the temperature jump, irrespective of the velocity slip. One may note that a grooved surface with trapped air, which exemplifies a superhydrophobic surface, amounts to the introduction of an insulating material. This is in sharp contrast to a finned surface, which amounts to the introduction a conducting material. The former will impede temperature rise, while the latter will enhance heat transfer.

5. The temperature jump has a positive effect on the flow rate in cases $\mathrm{I}(\mathrm{B})$ and $\mathrm{II}(\mathrm{B})$, where the temperature jump serves to prevent heat loss through the outer wall. In case $\mathrm{I}(\mathrm{B})$, the maximum flow rate, which occurs when the core radius is very small, is increased without bound by increasing either the velocity slip or the temperature jump. However, for a finite core radius, the flow rate in case $\mathrm{I}(\mathrm{B})$ will be independent of both slip and temperature jump when these two parameters are very large. In case II(B), the flow rate can be increased without bound by increasing the temperature jump, even for a finite core radius.

In this work, fully-developed flow is assumed, which may serve as an upper bound for finite lengths. It is desirable if the problem can be extended in a future study to developing natural convection in the entrance region of a channel of finite length. 


\section{Acknowledgments}

Financial support was given by the Research Grants Council of the Hong Kong Special Administrative Region, China, through Project No. HKU 715609E.

\section{References}

[1] Wang, C. Y., "Optimization of Natural Convection in Open Vertical Ducts with Heated Cores," Journal of Thermophysics and Heat Transfer, Vol. 24, 2010, pp. 669-672.

[2] Jaluria, Y., Natural Convection, Pergamon, Oxford, 1980.

[3] Joshi, H. M., "Fully Developed Natural Convection in an Isothermal Vertical Annular Duct," International Communications in Heat and Mass Transfer, Vol. 14, No. 6, 1987, pp. 657-664.

[4] El-Shaarawi, M. A. I., and Al-Nimr, M. A., "Fully Developed Laminar Natural Convection in Open-Ended Vertical Concentric Annuli," International Journal of Heat and Mass Transfer, Vol. 33, No. 9, 1990, pp. 1873-1884.

[5] Al-Nimr, M. A., "Analytical Solution for Transient Laminar Fully Developed FreeConvection in Vertical Concentric Annuli," International Journal of Heat and Mass Transfer, Vol. 36, No. 9, 1993, pp. 2385-2395.

[6] Ramammurthi, K., and Ravi, K., "Transient Natural Convection in a Cylindrical Annulus," Journal of Thermophysics and Heat Transfer, Vol. 11, No. 4, 1997, pp. 595-597.

[7] El-Shaarawi, M. A. I., Mokheimer, E. M. A., and Abulhamayel, H. I., "Limiting Values for Free-Convection Induced Flow Rates in Vertical Eccentric Annuli with an Isothermal Boundary," Numererical Heat Transfer, Part A, Applications, Vol. 39, No. 6, 2001, pp. $611-630$.

[8] Mokheimer, E. M. A., and El-Shaarawi, M., "Maximum Possible Induced Flow Rates in Open-Ended Vertical Eccentric Annuli with Uniform Heat Flux," International Journal of Numerical Methods for Heat and Fluid Flow, Vol. 15, No. 2, 2005, pp. 161-182. 
[9] Weng, H. C., and Chen, C. K., "Drag Reduction and Heat Transfer Enhancement Over a Heated Wall of a Vertical Annular Microchannel," International Journal of Heat and Mass Transfer, Vol. 52, 2009, pp. 1075-1079.

[10] Avci, M., and Aydin, O., "Mixed Convection in a Vertical Microannulus Between Two Concentric Microtubes," Journal of Heat Transfer, Vol. 131, 2009, p. 014502.

[11] Buonomo, B., and Manca, O., "Natural Convection Slip Flow in a Vertical Microchannel Heated at Uniform Heat Flux," International Journal of Thermal Sciences, Vol. 49, No. 8, 2010, pp. 1333-1344.

[12] Vinogradova, O. I., and Dubov, A. L., "Superhydrophobic Textures for Microfluidics," Mendeleev Communications, Vol. 22, 2012, pp. 229-236. 


\section{Captions}

Table 1 The maximum flow rate $Q_{\max }$ and the corresponding optimum core radius $b_{\mathrm{optm}}$ for some values of $\lambda$ and $\gamma$, including the cases shown in Figs. 4 and 5.

Figure 1 Definition sketch of the problem: fully-developed free convection in an openended vertical annular microchannel, with the inner wall maintained at either constant temperature or constant heat flux, and the outer wall maintained at ambient temperature. The core radius (normalized by the outer radius) is $b$, and the upward velocity is $w(r)$.

Figure 2 For case I (heating due to constant wall temperature), the flow rate (a) $Q_{\mathrm{I}}^{\mathrm{A}}$, and (b) $Q_{\mathrm{I}}^{\mathrm{B}}$, as a function of the slip length $\lambda$ and temperature jump coefficient $\gamma$, where $b=0.1$.

Figure 3 For case II (heating due to constant heat flux), the flow rate (a) $Q_{\mathrm{II}}^{\mathrm{A}}$, and (b) $Q_{\mathrm{II}}^{\mathrm{B}}$, as a function of the slip length $\lambda$ and temperature jump coefficient $\gamma$, where $b=0.2$.

Figure 4 Flow rate as a function of the core radius $b$ for the four cases: (a) $Q_{\mathrm{I}}^{\mathrm{A}}(b)$, (b) $Q_{\mathrm{I}}^{\mathrm{B}}(b),(\mathrm{c}) Q_{\mathrm{II}}^{\mathrm{A}}(b),(\mathrm{d}) Q_{\mathrm{II}}^{\mathrm{B}}(b)$, for $(\lambda, \gamma)=(0,0),(1,0),(0,1),(1,1)$.

Figure 5 The singular sharp rise of $Q_{\mathrm{I}}^{\mathrm{B}}$ as the core radius $b$ barely increases from zero, for $(\lambda, \gamma)=(0,0),(10,0),(0,10),(10,10)$. The crosses denote the peaks of the curves.

Figure 6 For the four cases, (a) the maximum flow rate $Q_{\max }$, and (b) the corresponding optimum core radius $b_{\mathrm{optm}}$, as functions of $\lambda$ or $\gamma$, where $\lambda=\gamma$. The inset in (a) is for a wider view of $Q_{\mathrm{I}, \max }^{\mathrm{B}}$ and $Q_{\mathrm{II}, \max }^{\mathrm{B}}$. 
Table 1: The maximum flow rate $Q_{\max }$ and the corresponding optimum core radius $b_{\mathrm{optm}}$ for some values of $\lambda$ and $\gamma$, including the cases shown in Figs. 4 and 5.

\begin{tabular}{c|c|c|c|c|c}
\hline$\lambda$ & $\gamma$ & $Q_{\mathrm{I}, \max }^{\mathrm{A}}\left(b_{\mathrm{optm}}\right)$ & $Q_{\mathrm{I}, \max }^{\mathrm{B}}\left(b_{\mathrm{optm}}\right)$ & $Q_{\mathrm{II}, \max }^{\mathrm{A}}\left(b_{\mathrm{optm}}\right)$ & $Q_{\mathrm{I}, \max }^{\mathrm{B}}\left(b_{\mathrm{optm}}\right)$ \\
\hline 0 & 0 & $0.05697(0.0947)$ & $0.05697(0.09479)$ & $0.01667(0.20954)$ & $0.01667(0.20954)$ \\
1 & 0 & $0.11151(0.18132)$ & $0.16657(0.07460)$ & $0.03741(0.25769)$ & $0.04681(0.20236)$ \\
0 & 1 & $0.01260(0.19311)$ & $0.10853(0.07951)$ & $0.01667(0.20954)$ & $0.05189(0.25591)$ \\
1 & 1 & $0.02777(0.24544)$ & $0.39492(0.06165)$ & $0.03741(0.25769)$ & $0.18098(0.25255)$ \\
\hline 10 & 0 & $0.12796(0.20688)$ & $0.42082(0.00935)$ & $0.04392(0.26875)$ & $0.08660(0.15923)$ \\
0 & 10 & $0.00161(0.20758)$ & $0.22501(0.01261)$ & $0.01667(0.20954)$ & $0.37484(0.27906)$ \\
10 & 10 & $0.00424(0.26749)$ & $3.92499\left(9.65 \times 10^{-6}\right)$ & $0.04392(0.26875)$ & $2.5668(0.21054)$ \\
\hline
\end{tabular}




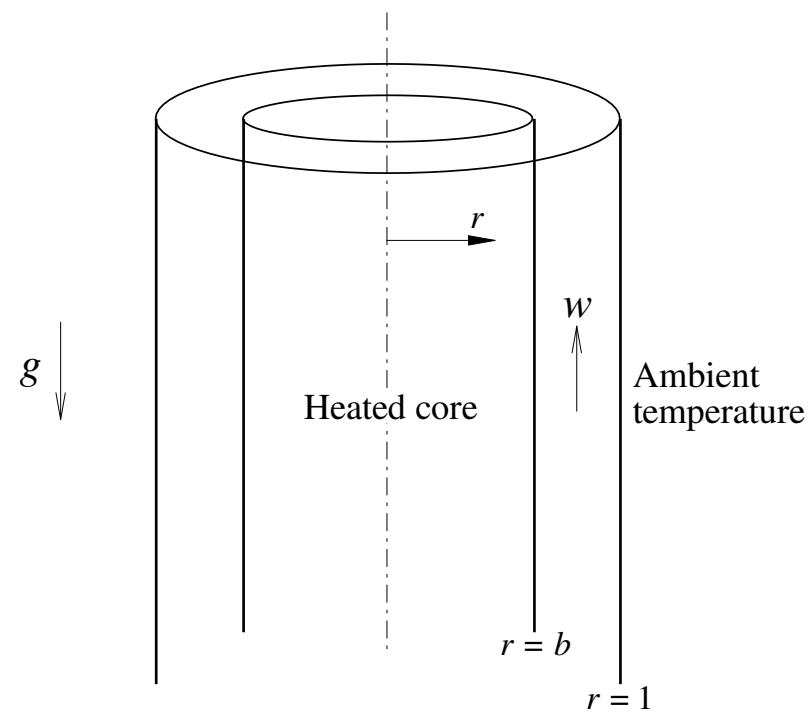

Figure 1: Definition sketch of the problem: fully-developed free convection in an open-ended vertical annular microchannel, with the inner wall maintained at either constant temperature or constant heat flux, and the outer wall maintained at ambient temperature. The core radius (normalized by the outer radius) is $b$, and the upward velocity is $w(r)$. 

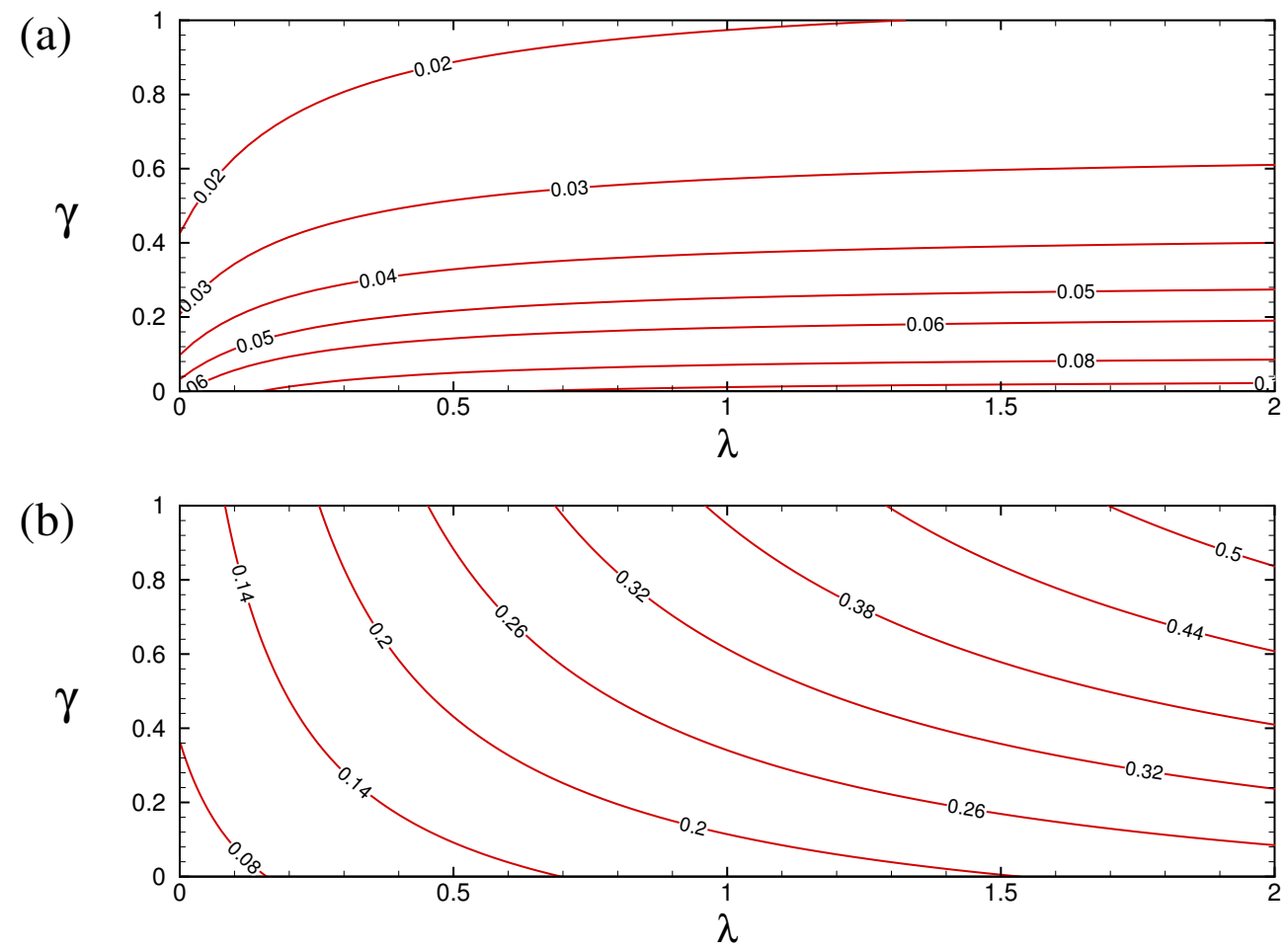

Figure 2: For case I (heating due to constant wall temperature), the flow rates (a) $Q_{\mathrm{I}}^{\mathrm{A}}$, and (b) $Q_{\mathrm{I}}^{\mathrm{B}}$, as functions of the slip length $\lambda$ and temperature jump coefficient $\gamma$, where $b=0.1$. 

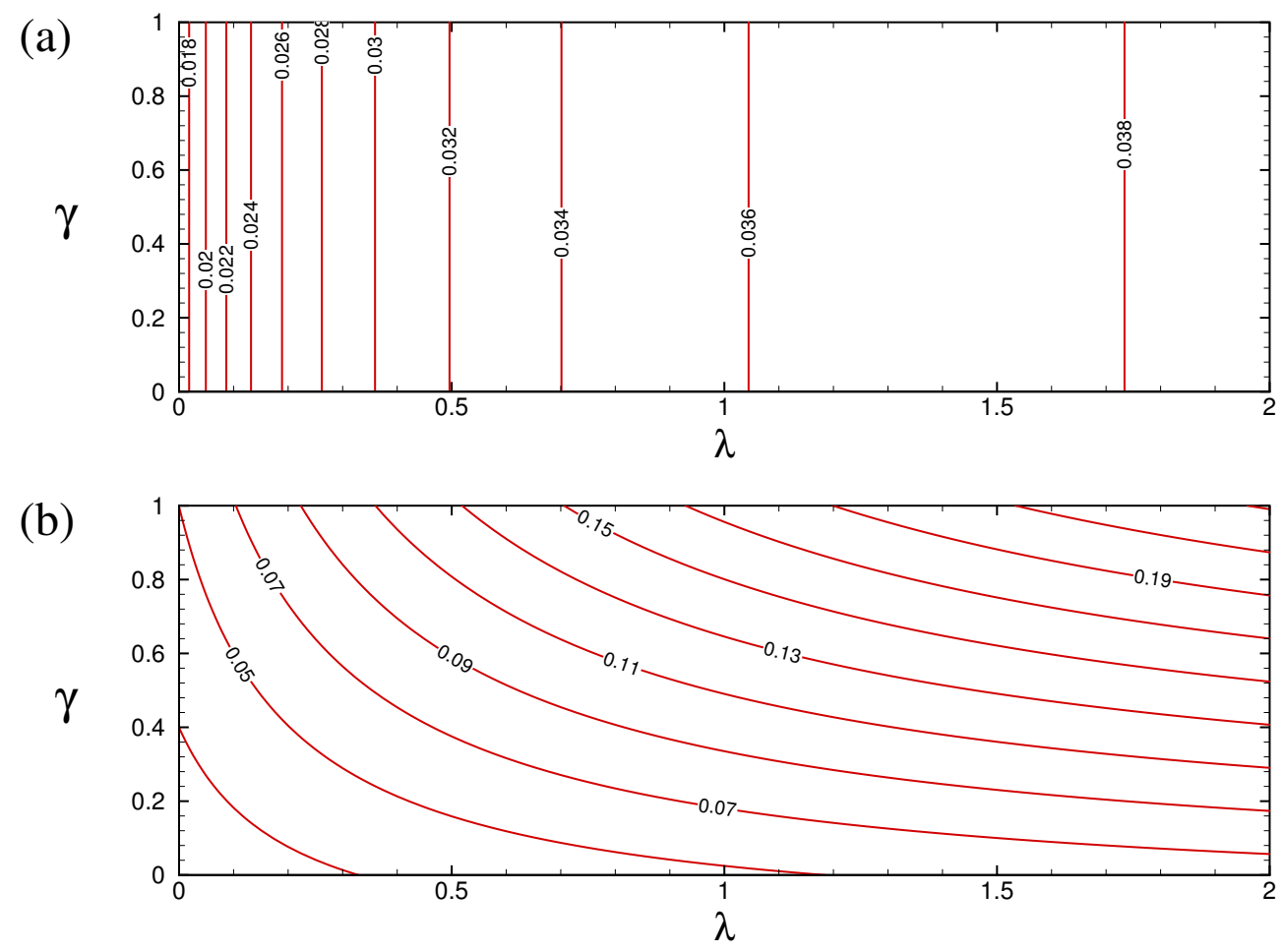

Figure 3: For case II (heating due to constant heat flux), the flow rates (a) $Q_{\mathrm{II}}^{\mathrm{A}}$, and (b) $Q_{\mathrm{II}}^{\mathrm{B}}$, as functions of the slip length $\lambda$ and temperature jump coefficient $\gamma$, where $b=0.2$. 
(a)

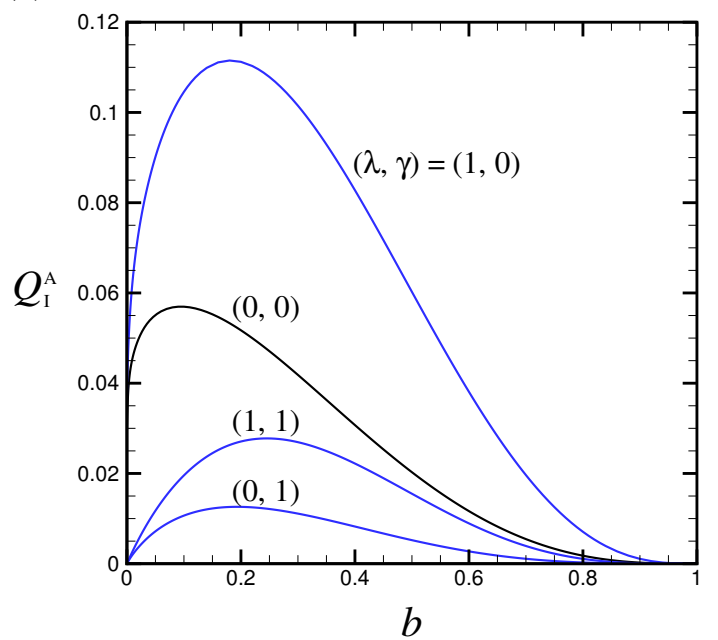

(c)

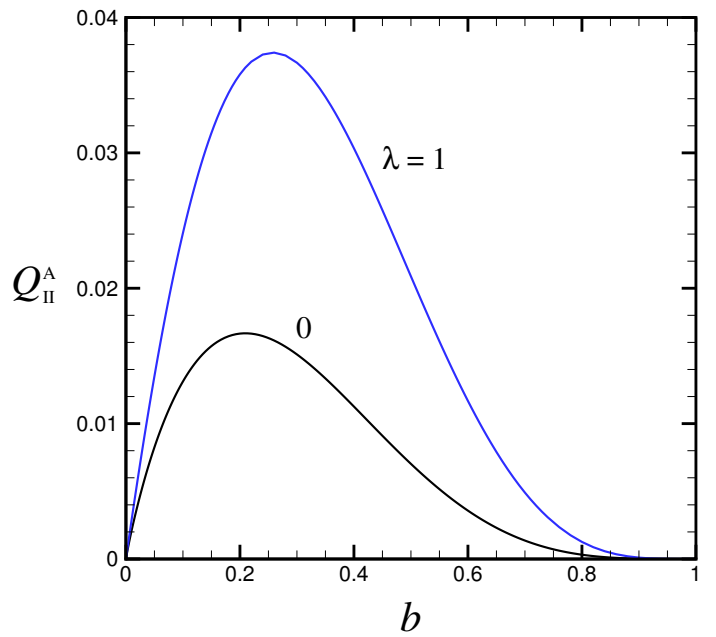

(b)

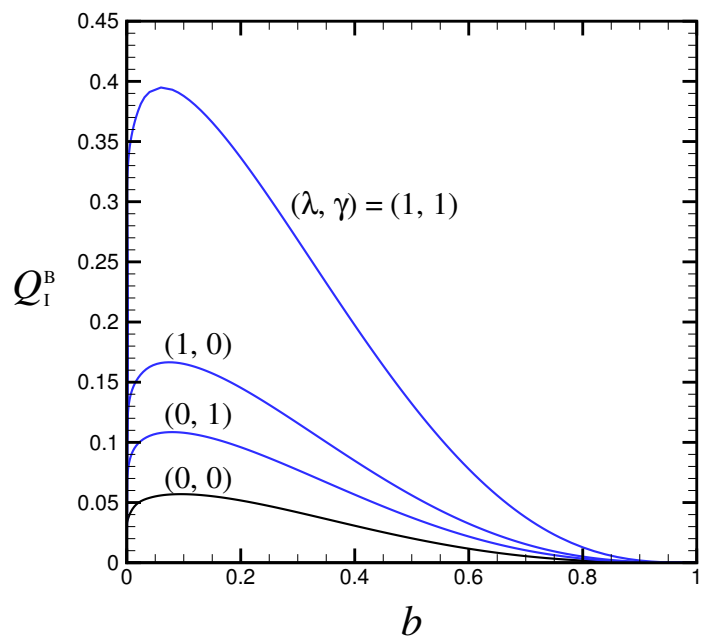

(d)

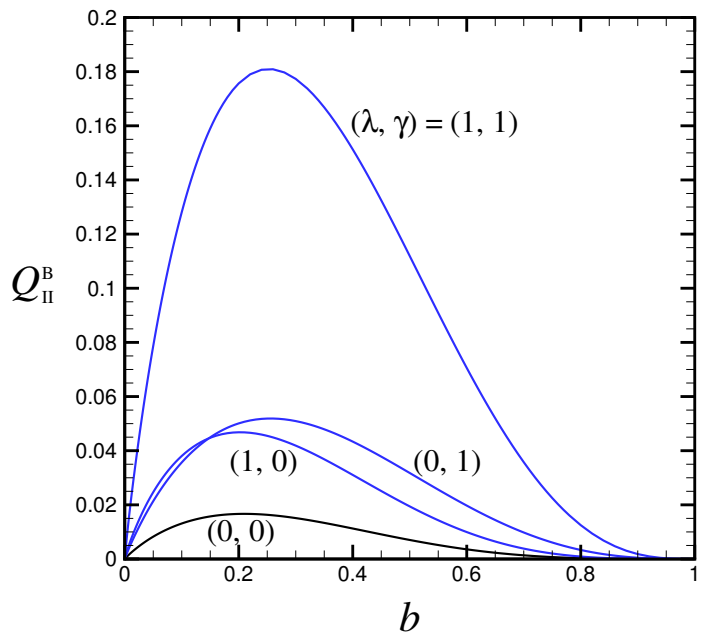

Figure 4: Flow rate as a function of the core radius $b$ for the four cases: (a) $Q_{\mathrm{I}}^{\mathrm{A}}(b),(\mathrm{b}) Q_{\mathrm{I}}^{\mathrm{B}}(b)$, (c) $Q_{\mathrm{II}}^{\mathrm{A}}(b),(\mathrm{d}) Q_{\mathrm{II}}^{\mathrm{B}}(b)$, for $(\lambda, \gamma)=(0,0),(1,0),(0,1),(1,1)$. 


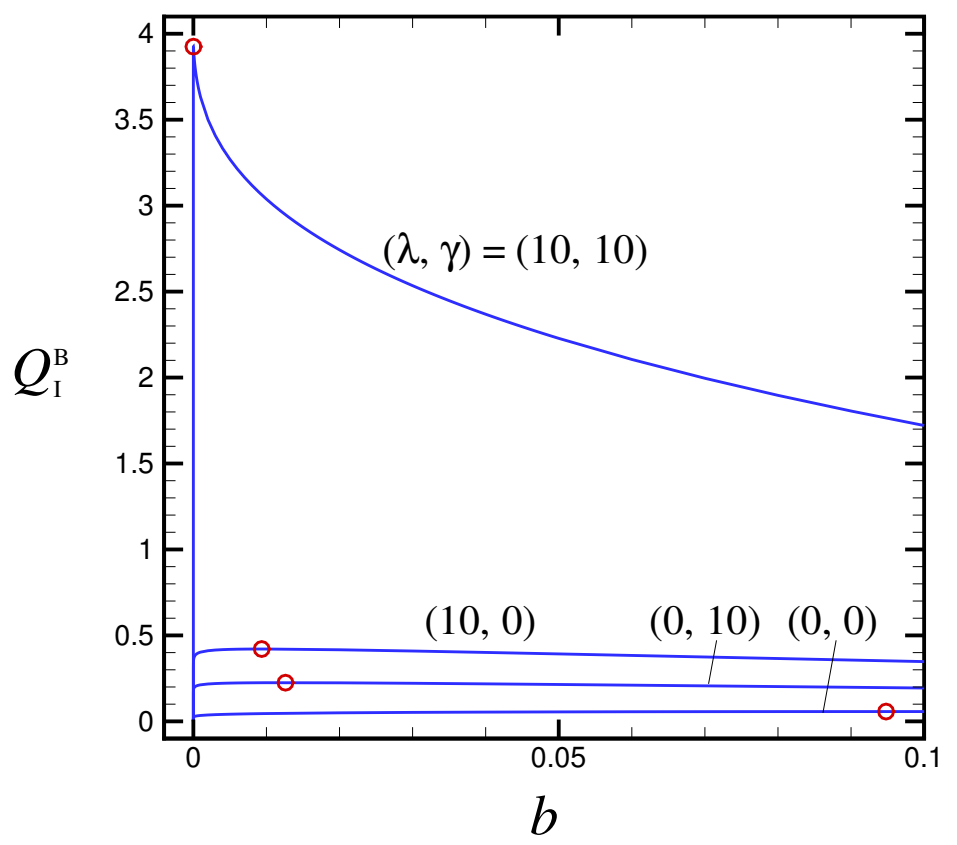

Figure 5: The singular sharp rise of $Q_{\mathrm{I}}^{\mathrm{B}}$ as the core radius $b$ barely increases from zero, for $(\lambda, \gamma)=(0,0),(10,0),(0,10),(10,10)$. The circles denote the peaks of the curves. 
(a)

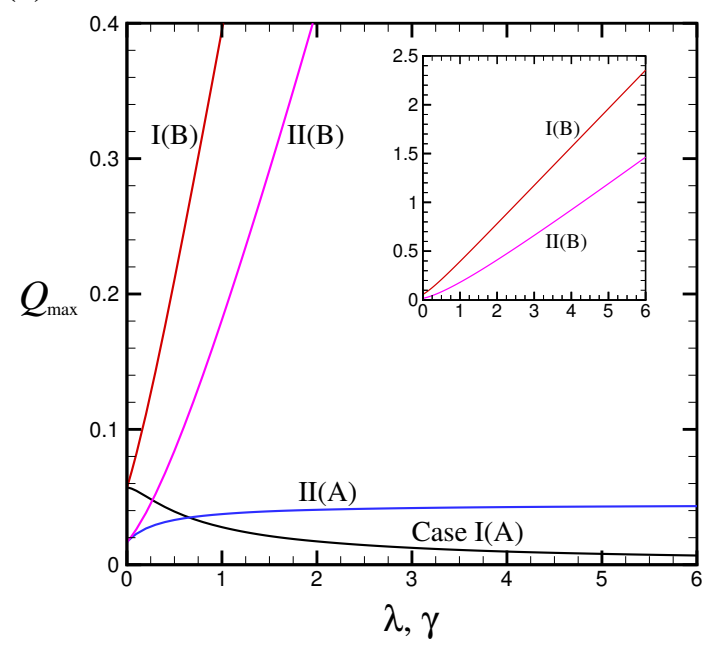

(b)

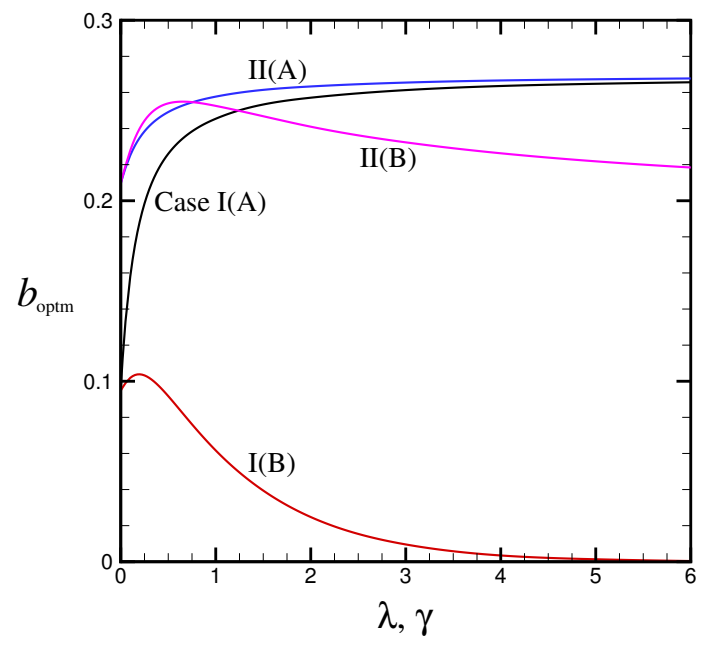

Figure 6: For the four cases, (a) the maximum flow rate $Q_{\max }$, and (b) the corresponding optimum core radius $b_{\text {optm }}$, as functions of $\lambda$ or $\gamma$, where $\lambda=\gamma$. The inset in (a) is for a wider view of $Q_{\mathrm{I}, \max }^{\mathrm{B}}$ and $Q_{\mathrm{II}, \max }^{\mathrm{B}}$. 